\title{
KONTRASTIVER VERGLEICH DES DEUTSCHEN UND SERBISCHEN WORTBILDUNGSSYSTEMS IM BEREICH DER ZUSAMMENGESETZTEN UND SUFFIGIERTEN PERSONENBEZEICHNUNGEN
}

\author{
MihaEla LALiĆ
}

\section{Wortbildung von Personenbezeichnungen im Deutschen}

Hinsichtlich der morphologischen Struktur von Personenbezeichnungen im Deutschen kann festgestellt werden, dass es sich bei den meisten Lexemen um Zusammensetzungen und Ableitungen handelt.

Peter Braun (1997: 54) hat eine Untersuchung zum Thema Personenbezeichnungen im Deutschen vorgenommen und als Korpus diente das Deutsche Universalwörterbuch, in dem etwa 15.000 Personenbezeichnungen enthalten sind. Auf der Basis der 10300 Personenbezeichnungen hat Braun nur 8\% der Simplizia festgestellt und 92\% stellen dagegen Wortbildungskonstruktionen, vorwiegend Zusammensetzungen und Ableitungen, dar.

$\mathrm{Zu}$ den Simplizia gehören meistens Wörter aus dem Bereich der Verwandschaftszbezeichnungen wie Bruder, Vater, Neffe und nicht wenige neue Simplizia sind Entlehnungen aus dem Englischen wie Baby, Freak u. a.

Im Folgenden sollen Personenbezeichnungen als Produkte der deutschen Wortbildung beschrieben werden. 


\subsection{Kompositionelle Bildungen}

Im Deutschen lassen sich determinative (Allgemeinarzt, Fahrradfahrer, Putzfrau)und kopulative personale Komposita (Dichterkomponist, SchauspielerRegisseur, Waisenkind) feststellen.

Unter Determinativkomposita versteht man Bildungen, bei denen das Grundwort durch das Bestimmungswort modifiziert bzw. näher bestimmt wird. Bei den Kopulativkomposita besteht, im Gegensatz zu den Determinativkomposita, kein Unterordnungsverhältnis. Die Glieder der Kopulativkomposita gehören zu derselben Wortart, stehen in einem koordinierenden Verhältnis zueinander und beziehen sich auf das gleiche Denotat, wobei sie jeweils unterschiedliche Eigenschaften dessen hervorheben bzw. es aus unterschiedlichen Perspektiven beschreiben.

\subsubsection{Possessivkomposita}

Es gibt im Deutschen eine besondere Gruppe der Komposita, nämlich die sog. Possessivkomposita. Sie werden in der Literatur entweder als Sonderfall der Determinativkomposita angesehen oder als ein besonderer Kompositionstyp. Nach ihrer Konstituentenstruktur handelt es sich einerseits um Adjektiv-NomenKomposita wie Schlaukopf, Dickbauch, Rothaut und andererseits um NomenNomen-Komposita wie Hasenfuß, Milchbart, Spitzbart. Bildungen mit verbalem Erstglied wie Hinkebein oder Schielauge sind selten (Pittner 1996: 179).

Es gibt zahlreiche Nominalkomposita wie Spitzbart, Dickbauch, Langbein, Milchgesicht, bei denen beide Lesarten möglich sind. Dies kann an folgenden Beispielen dargestellt werden (Olsen 1990: 143):

- Sein Krauskopf blockiert die Sicht zur Leinwand.

- Der Krauskopf lässt sich hier nicht wieder sehen.

Wenn sich das Nominalkompositum Krauskopf auf einen krausen Kopf wie im ersten Satz bezieht, so stellt es ein (endozentrisches) Determinativkompositum dar. Wird es aber nach dem Pars-Pro-Toto-Prinzip wie im zweiten Satz verwendet, um einen Menschen mit dieser Eigenart zu benennen, so liegt ein (exozentrisches) Possessivkompositum vor, also eine Personenbezeichnung.

Die Eigenart solcher Bildungen, die eine exozentrische Struktur aufweisen, besteht aber nicht in ihren strukturellen Eigenschaften, denn sie sind von der Struktur her identisch mit endozentrischen Strukturen, sondern sie besteht lediglich in der Sprachverwendung (Olsen 1990: 143). Genau wie bei (endozentrischen) Determinativkomposita ist auch bei den (exozentrischen) Possessivkomposita das Letztglied der formale Kopf der gesamten Konstruktion. 
Dies gilt ausnahmslos und geht sogar so weit, dass es zu Widersprüchen zwischen dem Genus des Kompositums und dem natürlichen Geschlecht der damit bezeichneten Person kommen kann. So sind Wörter wie Hinkebein oder Rothaut immer Neutrum bzw. feminin (wie Bein und Haut), unabhängig davon, ob mit ihnen Frauen oder Männer bezeichnet werden. Interessanterweise enthält auch das Kompositum Blaustrumpf, das sich lediglich auf Frauen bezieht, ein maskulines Genus, weil das Letztglied maskulin ist (Pittner 1996: 182).

Possessivkomposita gehören teilweise in die deutsche Umgangssprache, sind expressiv und ausdrucksvoll. Diese Bildungen sind vor allem mit Grundwörtern zu Körper-, Kleider- und Pflanzenteilen oder Tiernamen entstanden,wie zum Beispiel:Trotzkopf, Plappermaul, Flattergeist, Spaßvogel, Angsthase, Brummbär, Bücherwurm, Plaudertasche, Federfuchs, Grünschnabel, Dummkopf, Geizhals, Langfinger, Rothaut u.a.

\subsubsection{Bildungen mit Affixoiden}

Wenn bestimmte Konstituenten der Komposita stark reihenbildend auftreten, bekommen sie allmählich den Präfix- bzw. Suffixcharakter. Häufig vorkommende Präfixoide, die vorwiegend mit Personenbezeichnungen verbunden sind, sind: Dreck-, Mist-, Sau- Teufels- und Erz-. Die Funktion des Präfixoids besteht oft darin, zusammen mit neutralen Lexemen neue pejorative Lexeme zu bilden wie Dreckmensch, Mistkerl, Saukerl, Teufelskerl, Erzlügner u. a. Pejorative Präfixoide und pejorative Lexeme werden auch kombiniert. Das Präfixoid verstärkt in diesem Fall die Bedeutung des pejorativen Lexems, wie in Drecksau, Sauluder, Drecksack, oder Sauvieh. Pejorativen Lexemen werden auch neutrale Präfixoide zugefügt und hier spielen Präfixoide die Rolle des Intensifikators, wie in den Lexemen Unterschwein, Vollidiot, Riesennull und Außentrottel (Havryliv 2003: 43).

Eine besondere Eigenart des Deutschen ist die Personenbezeichnung unter Verwendung eines Vor- oder Familiennamens als zweiter Konstituente, häufig vor allem in Mundarten und der Umgangssprache. Aus Wortgruppen, oft mit Adjektiv und Substantiv, z.B. der ängstliche, kräftige, schlaue Meier entstehen Personenbezeichnungen wie Angst-, Kraft-, und Schlaumeier oder es werden Verbalstamm und gekürzte Form des Vor- und Familiennamens zusammengezogen wie in Heulsuse oder Heulpeter. Häufig vorkommende Konstituente sind folgende:

$\begin{array}{llll}\text {-hans } & \rightarrow \text { Prahlhans } & \text {-michel } & \rightarrow \text { Quatschmichel } \\ \text {-liese } & \rightarrow \text { Bummelliese } & \text {-philipp } & \rightarrow \text { Zappelphilipp } \\ \text {-fritze } & \rightarrow \text { Nörgelfritze } & \text {-berger } & \rightarrow \text { Drückeberger } \\ \text {-peter } & \rightarrow \text { Lügenpeter } & \text {-bruder } & \rightarrow \text { Zechbruder }\end{array}$




$$
\begin{array}{llll}
\text {-heini } & \rightarrow \text { Trödelheini } & \text {-schwester } & \rightarrow \text { Betschwester } \\
\text {-hanne } & \rightarrow \text { Klatschhanne } & \text {-onkel } & \rightarrow \text { Reiseonkel }
\end{array}
$$

Eine Gegenüberstellung solcher Wörter mit männlichen und weiblichen Vornamen zeigt, dass durch das zweite Element (Namen) das Geschlecht differenziert wird.

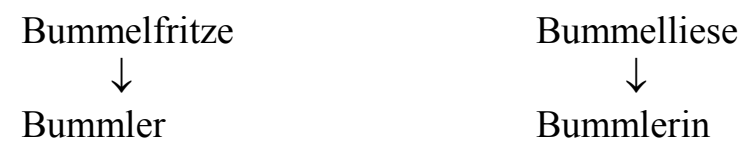

Wird ein Mann mit einem weiblichen Namen bezeichnet, so wirkt die Personenbezeichnung expressiver und wird stärker empfunden. Die zweite Konstituente kann in gewissen Grenzen variieren, die lexikalische Bedeutung des Ganzen bleibt dabei jedoch unveränderlich, wie man es bei den Lexemen Heulsuse und Heulliese sehen kann. Der semantische Kern ist in der ersten Konstituente eingeschlossen, die meist die Form eines Verbalstamms aufweist. Die starke Expressivität der ersten Konstituente bedingt im großen Maße die Anschaulichkeit und Emotionalität des Wortes im Ganzen.

Die zweiten Konstituenten verhalten sich wie Suffixe, sie haben eine abstraktere Bedeutung, sind reihenbildend, haben keine lexikalische Bedeutung und differenzieren das Geschlecht. Man könnte die zweiten Konstituenten als Suffixe bezeichnen, jedoch sind sie keine neutralen Suffixe, wie beispielsweise -er, sondern emotional gefärbte Wortelemente in Substantiven mit starkem expressivem Gehalt.

\subsubsection{Zusammenbildungen}

Zusammenbildungen sind eine in der Forschungsliteratur umstrittene Klasse von Bildungen, die unterschiedlich eingeordnet werden. Einerseits werden sie als Mischphänomen der Komposition und Derivation und andererseits als Phrasenderivate betrachtet. Im Unterschied zu Komposita, die aus mindestens zwei lexikalischen Einheiten bestehen, stellt das zweite Glied vieler dieser Bildungen wie Wichtigtuer keine eigenständige lexikalische Einheit dar. Sie ist ein Derivat, das aus der Basis $t u$ - und dem Wortbildungssuffix -er besteht. Die wichtigsten

\begin{tabular}{|c|c|c|c|c|c|}
\hline -macher & $\rightarrow$ & Filmemacher & -steller & $\rightarrow$ & Fragesteller \\
\hline -haber & $\rightarrow$ & Liebhaber & -tuer & $\rightarrow$ & Nichtstuer \\
\hline -halter & $\rightarrow$ & Rekordhalter & -brecher & $\rightarrow$ & Gesetzesbrecher \\
\hline -gänger & $\rightarrow$ & Fußgänger & -binder & $\rightarrow$ & Buchbinder \\
\hline -folger & $\rightarrow$ & Thronfolger & & & \\
\hline
\end{tabular}
Formtypen im Bereich von Personenbezeichnungen: 


\subsubsection{Zusammenrückungen oder Satzwörter}

Exozentrische Bildungen wie Trautsichnichts, Störenfried und Springinsfeld werden unterschiedlich eingeordnet, jedoch sind sich die meisten Autoren einig, dass sie sich wegen einiger typischer Aspekte ihrer morphologischen Struktur vom normalen Kompositionsmuster abheben. Sie werden als Reanalysen und Lexikalisierungen von eindeutig phrasalen Sequenzen angesehen. Typisch für viele dieser Bildungen ist, dass sie ein charakteristisches Merkmal bzw. Verhalten von Wesen, vor allem eines Menschen, bezeichnen, die metonymisch oder synekdochisch als Benennung für diese Wesen selbst (exozentrisch) verwendet werden (Zdzislaw 1999: 11). Typische Zusammenrückungen im Deutschen sind: Dreikäsehoch, Garnichts, Gernegroß, Gerneklug, Möchtegern, Nimmersatt u.a.

\subsection{Suffixale Bildungen}

Es gibt eine ganze Reihe von Suffixen, mit deren Hilfe aus Nomen, Verben und Adjektiven neue Personenbezeichnungen entstehen. Die folgenden Suffixe bezeichnen vor allem Personen, die durch das von der Basis Bezeichnete semantisch näher bestimmt werden (Donalies 2005: 104-105):

\begin{tabular}{|c|c|c|c|}
\hline \multicolumn{2}{|c|}{$\begin{array}{l}\text { DENOMINALE } \\
\text { SUBSTANTIVE }\end{array}$} & $\begin{array}{l}\text { DEVERBALE } \\
\text { SUBSTANTIVE }\end{array}$ & $\begin{array}{l}\text { DEADJEKTIVISCHE } \\
\text { SUBSTANTIVE }\end{array}$ \\
\hline $\begin{array}{l}\text {-er (Handwerker) } \\
\text {-ler (Tischler) } \\
\text {-ner (Rentner) } \\
\text {-ier (Hotelier) } \\
\text {-är (Funktionär) } \\
\text {-ar (Bibliothekar) } \\
\text {-ant/-ent (Asylant) } \\
\text {-ist (Rassist) }\end{array}$ & $\begin{array}{l}\text {-e (Philologe) } \\
\text {-aner (Republikaner) } \\
\text {-ling (Dichterling) } \\
\text {-and (Doktorand) } \\
\text {-iker (Alkoholiker) } \\
\text {-bold (Witzbold) } \\
\text {-in (Professorin) } \\
\text {-i (Mutti) }\end{array}$ & $\begin{array}{l}\text {-er (Schwimmer) } \\
\text {-eur (Kontrolleur) } \\
\text {-euse (Masseuse) } \\
\text {-ant (Demonstrant) } \\
\text {-ent (Produzent) } \\
\text {-ling (Prüfling) } \\
\text {-ator (Organisator) } \\
\text {-bold (Raufbold) }\end{array}$ & $\begin{array}{l}\text {-ling (Rohling) } \\
\text {-ian/jan (Grobian) } \\
\text {-i (Blondi) } \\
\text {-iker (Fanatiker) } \\
\text {-o (Brutalo) } \\
\text {-ine (Blondine) } \\
\text {-chen (Dummchen) }\end{array}$ \\
\hline
\end{tabular}

\section{Wortbildung von Personenbezeichnungen im Serbischen}

Hinsichtlich der morphologischen Struktur von Personenbezeichnungen kann festgestellt werden, dass es sich bei den meisten Lexemen um Ableitungen und Zusammensetzungen handelt, wobei die Ableitungen deutlich überwiegen. Im Folgenden sollen Personenbezeichnungen als Produkte der serbischen Wortbildung beschrieben werden. 


\subsection{Kompositionelle Bildungen}

In der serbischen Forschungstradition zählen zu der Komposition zwei Wortbildungsprozesse. Auf der einen Seite gibt es die sog. "reine" Zusammensetzung Zusammenfügung von zwei eigenständigen lexikalischen Stammformen wie im Beispiel romanopisac 'Romanschriftsteller', in dem das Nomen pisac 'Schriftsteller', das vom Verb pisati 'schreiben' und dem Suffix - $a c$ abgeleitet ist, ein eigenständiges Lexem darstellt (Barić 1980: 27). Endozentrische personale Komposita dieser Art sind im Serbischen nicht produktiv und bei vielen handelt es sich um Lehnübersetzungen aus dem Deutschen. Einige Beispiele sind:

$$
\begin{aligned}
\text { zemljoposednik 'Grundbesitzer' } \rightarrow & \text { zusammengesetzt aus zemlja 'Grundstück' } \\
& \text { und posednik 'Besitzer, Eigentümer' } \\
\text { prestolonaslednik 'Thronfolger' } \rightarrow & \text { zusammengesetzt aus presto 'Thron' und } \\
& \text { naslednik'Erbe, Nachfolger' } \\
\text { vojskovođa 'Heerführer' } \rightarrow & \text { zusammengesetzt aus vojska 'Heer' und } \\
& \text { vođa 'Führer' }
\end{aligned}
$$

Neben den genannten Komposita, gibt es auch Zusammensetzungen von zwei Stammformen mit der gleichzeitigen Suffigierung oder impliziter Ableitung des Letztglieds. Das Letztglied fungiert nicht als eine eigenständige lexikalische Einheit. Das kann am Beispiel staklorezac 'Glaser' veranschaulicht werden, das aus staklo 'Glas' und rezac (abgeleitet vom Verb rezati 'schneiden, schnitzen' und dem Suffix -ac) besteht.

Rezac stellt im Serbischen kein eigenständiges Lexem dar und Bildungen dieser Art sind von der Form her vergleichbar mit den Zusammenbildungen des Typs Wichtigtuer im Deutschen, deren Letztglieder ebenfalls keine eigenständigen lexikalischen Einheiten darstellen. Lexeme dieser Art sehen wie folgt aus:

$$
\begin{aligned}
\text { vatrogasac 'Feuerwehrmann' } \rightarrow & \text { zusammengesetzt aus vatra 'Feuer' und } \\
& \text { gasac (abgeleitet vom Verb gasiti 'löschen' } \\
& \text { und dem Suffix -ac) } \\
\text { knjigovezac 'Buchbinder' } \rightarrow & \text { zusammengesetzt aus knjiga 'Buch' und } \\
& \text { vezac (abgeleitet vom Verb vezati 'binden' } \\
& \text { und dem Suffix -ac) } \\
\text { Machthaber 'vlastodržac' } \rightarrow & \text { zusammengesetzt aus vlast 'Herrschaft, } \\
& \begin{array}{l}
\text { Macht' und držac (abgeleitet vom Verb } \\
\text { držati 'halten, festhalten' und dem Suffix -ac) }
\end{array}
\end{aligned}
$$

Außer zwei genannten endozentrischen Gruppen von personalen Komposita, gibt es im Serbischen auch exozentrische Personenbezeichnungen, die meist in spöttisch-ironischer Weise auf die Tätigkeit oder das Verhalten eines Menschen 
anspielen (Klajn 2002: 35). Diese Bildungen gehören zu der Gruppe der sog. Imperativkomposita und können anhand von folgenden Beispielen dargestellt werden:

Gulikoža 'Leuteschinder' (wortwörtlich: Schind (IMPER.) die Haut (NOM)) Palikuća 'Brandstifter' (wortwörtlich: Brenn (IMPER.) das Haus (NOM)) Vucibatina 'Landstreicher' (wortwörtlich: Zieh (IMPER.) den Prügel (NOM)) Vrtirep 'Zappelphilipp' (wortwörtlich: Dreh (IMPER.) den Schwanz (NOM)) Mutivoda 'Intrigant' (wortwörtlich: Trübe (IMPER.) das Wasser (NOM))

\subsection{Suffixale Bildungen}

Das Serbische bietet eine ganze Fülle von Suffixen für Personenbezeichnungen von verbaler Basis und noch größer ist die Zahl der Suffixe, wo Personenbezeichnungen von substantivischen und adjektivischen Basen gebildet werden. Wortbildungsprozesse sind im Allgemeinen sehr komplex für die Wortbildungsanalyse des Serbischen, weil infolge morphophonemischer Änderungen die genaue Morphemgrenze nicht mehr erkennbar ist. Hier gehen sogar die Meinungen der Fachleute auseinander, wo bei bestimmten Bildungen die Morphemgrenze zu setzen ist.

Hier ist eine Übersicht von Suffixen:

\begin{tabular}{|l|l|l|l|}
\hline \multicolumn{2}{|c|}{ DENOMINALE SUBSTANTIVE } & \multicolumn{1}{c|}{$\begin{array}{c}\text { DEVERBALE } \\
\text { SUBSTANTIVE }\end{array}$} & $\begin{array}{c}\text { DEADJETIVISCHE } \\
\text { SUBSTANTIVE }\end{array}$ \\
\hline -a (profa) & -džija (kamiondžija) & -(a)c (borac) & -(a)c (stranac) \\
-(a)c (omladinac) & -aš (odbojkaš) & -ar (slikar) & -ik (srećnik) \\
-ar (ribar) & -er (fudbaler) & -ač (predavač) & -ica (pijanica) \\
-ica (učiteljica) & -ijer (finansijer) & -lac (spasilac) & -ist(a) (humanista) \\
-ka (ministarka) & -in (Bugarin) & -nik (izvoznik) & -ajlija (novajlija) \\
-(n)ik (državnik) & -inja (junakinja) & -telj (staratelj) & -(a)k (ludak) \\
-ist(a) (vaterpolista) & -ionar/-ioner & -av(a)c (lajavac) & -an (velikan) \\
-ov(a)c/-ev(a)c & (misionar) & -aroš (pecaroš) & -aš (bogataš) \\
(oskarovac) & -ić /-čić (đačić) & -ator (gnjavator) & -enik (sveštenik) \\
-jak (seljak) & -ičar (hemičar) & -adžija (šaljivdžija) & -in (domaćin) \\
-al(a)c (gimnazijalac) & -kinja (favoritkinja) & -ent (student) & -ić (slabić) \\
-anac (Afrikanac) & -or (atentator) & -er (programer) & -kinja (crnkinja) \\
-anin/-janin & -onja (glavonja) & -iša (štediša) & -onja (mlakonja) \\
(Beograđanin) & -uša (prostakuša) & -lica (lutalica) & -uša (plavuša) \\
-aroš (džeparoš) & -lija (zanatlija) & -lo (džangrizalo) & -ko (trapavko) \\
-ator (snagator) & -če (jedinče) & -uša (smrduša) & -al(a)c (profesionalac) \\
\hline
\end{tabular}

Eine Besonderheit der serbischen Sprache stellen die Substantive des gemeinsamen Geschlechts dar, die gleichzeitig als Bezeichnung für einen Mann und eine Frau gebraucht werden (in einer entsprechenden Situation). Einige dieser Beispiele sind (Stanojčić 2010: 217): 


$$
\begin{array}{ll}
\text { propalica } & \rightarrow \text { Liederjan } \\
\text { piskaralo } & \rightarrow \text { Federfuchser, Schreiberling } \\
\text { neznalica } & \rightarrow \text { Nichtwisser } \\
\text { ulizica } & \rightarrow \text { Schmeichler }
\end{array}
$$

Viele dieser Substantive wirken sehr expressiv und gehören größtenteils in die serbische Umgangssprache. Ungewöhnlich reich sind die Möglichkeiten, die mit Hilfe der Suffixe der subjektiven Einschätzung geschaffen werden. Hier können die verschiedensten feinsten Bedeutungsschattierungen ausgedrückt werden. Es werden drei große Gruppen von Suffixen der subjektiven Einschätzung unterschieden:

$\begin{array}{lll}\text { Die Verkleinerungssuffixe (Diminutiva): } & \text { 'Dine kleine Frau' } \\ \text {-ica: ženica } & \rightarrow & \text { 'ein kleines Kind' } \\ \text {-ence: detence } & \rightarrow & \text { 'ein keines }\end{array}$

Die pejorativen Suffixe:

-etina: ženetina $\rightarrow$ 'Frauenzimmer, Weib'

-ura: pijandura $\rightarrow$ 'Trunkenbold'

-erda: gluperda $\rightarrow$ 'Vollidiot'

Die Vergrößerungssuffixe (Augmentativa):

-onja: glavonja $\rightarrow$ 'Großkopferte'

-ina: momčina $\rightarrow$ 'ein ganzer Kerl'

\section{Deutsche und serbische Personenbezeichnungen im Vergleich}

Aus der Konfrontation einer großen Zahl der Personenbezeichnungen im Deutschen und im Serbischen ergibt sich folgendes:

Beide Sprachen verfügen über außerordentlich reiche Wortbildungsmöglichkeiten im Bereich von Personenbezeichnungen. In keiner anderen Substantivgruppe gibt es derartig viele Wortbildungsmuster und die Konfrontation der Ausdrucksmittel und Wortbildungsmöglichkeiten in beiden Sprachen zeigt, dass diese verschieden verteilt sind, d.h. ein Teil der Wortbildungsmuster überwiegt, ist mehr verbreitet in einer der Sprachen oder fehlt ganz. Während im Serbischen die suffixale Ableitung eine außerordentliche Rolle bei der Bereicherung des Wortschatzes, besonders im Bereich von Personenbezeichnungen, spielt, zeichnet sich die deutsche Sprache durch eine große Zahl von Zusammensetzungen aus. Das im Serbischen stark entwickelte System von Suffixen steht einer bedeutend geringeren Zahl von Suffixen im Deutschen gegenüber. Für die Wiedergabe der vielfältigen Suffixe im Serbischen, benutzt die deutsche Sprache sowohl Suffixe, die dieselbe Funktionen wie die serbischen Suffixe ausüben können, als 
auch andere Möglichkeiten der Wortbildung. Nur bei Ableitungen von fremdsprachiger Basis besteht in beiden Sprachen eine völlige Übereinstimmung:

$$
\begin{array}{ll}
\text { er } \rightarrow \text { ar } & \\
\text { Komiker } & \rightarrow \text { komičar } \\
\text { Kritiker } & \rightarrow \text { kritičar } \\
\text { Musiker } & \rightarrow \text { muzičar } \\
\text { Alkoholiker } & \rightarrow \text { alkoholičar }
\end{array}
$$

Den verschiedenen serbischen Suffixen entsprechen im Deutschen auch verschiedene Suffixe. Man kann jedoch gewisse Entsprechungen der Suffixe feststellen. So werden im Serbischen deverbale Bildungen auf -lac fast ausnahmslos mit deutschen Ableitungen auf -er übersetzt:

$$
\begin{array}{ll}
\text { prevodilac } & \rightarrow \text { Übersetzer, vom Verb prevoditi 'übersetzen' } \\
\text { stvaralac } & \rightarrow \text { Schöpfer, vom Verb stvarati 'schöpfen' } \\
\text { mislilac } & \rightarrow \text { Denker, vom Verb misliti 'denken' } \\
\text { branilac } & \rightarrow \text { Verteidiger, vom Verb braniti 'verteidigen' }
\end{array}
$$

Deadjektivische Substantive auf $-a c$ werden im Deutschen durch substantivierte Adjektive wiedergegeben:

$\begin{array}{lll}\text { stranac } & \rightarrow & \text { Fremder, von adjektivischer Basis stran- 'fremd' } \\ \text { mudrac } & \rightarrow & \text { Weiser, von adjektivischer Basis mudr- 'weise' } \\ \text { svetac } & \rightarrow & \text { Heiliger, von adjektivischer Basis svet- 'heilig' }\end{array}$

Serbische Ableitungen von substantivischer Basis haben im Deutschen oft das Kompositum, bzw. die Zusammenbildung als Entsprechung:

$$
\begin{array}{ll}
\text { zubar } & \rightarrow \text { Zahnarzt, vom Substantiv zub 'Zahn' } \\
\text { dimnjičar } & \rightarrow \text { Schornsteinfeger, vom Substantiv dimnjak 'Schornstein' } \\
\text { grobar } & \rightarrow \text { Totengräber, vom Substantiv grob 'Grab' }
\end{array}
$$

Die größte Diskrepanz weisen deutsche und serbische expressive Personenbezeichnungen auf. Beide Sprachen sind außerordentlich reich an expressiven Personenbezeichnungen. Wie unterschiedlich aber die Expressivität in beiden Sprachen zum Ausdruck gebracht wird, beweist die Gegenüberstellung der Wortbildungsmodelle dieser Sprachen. So haben deutsche expressive Personenbezeichnungen mit Namen oder Vornamen als zweiter Konstituente keinen entsprechenden Platz im Serbischen, denn diesen Wortbildungstyp gibt es im Serbischen nicht. Im Gegensatz zum Deutschen zeichnen sich serbische Personenbezeichnungen vor allem durch eine größere Breite der Motivation der Suffixableitungen mit subjektiver Einschätzung aus.

Die untersuchten Beispiele zeigen, dass den meisten deutschen expressiven Personenbezeichnungen serbische Suffixableitungen mit verschiedenen Suffixen 
entsprechen (vor allem die der subjektiven Einschätzung), sowie Imperativkomposita, die jedoch im modernen Serbischen nicht produktiv sind.

Ein Teil der serbischen Substantive mit den Suffixen der subjektiven Einschätzung wird im Deutschen wiedergegeben durch:

Deonymische Personenbezeichnungen: plačljivica $\rightarrow$ Heulsuse zu plakati 'weinen'

Metaphorische Bildungen:

gunđalo $\rightarrow$ Brummbär zu gunđati 'brummen'

Bildungen mit dem Präfixoid: $\quad$ vragolan $\rightarrow$ Teufelskerl zu vrag 'Teufel'

Possessivkomposita: raščupanko $\rightarrow$ Strubbelkopf zu raščupan 'zerzaust'

\section{Zusammenfassung}

Anhand einer Untersuchung von über 2000 Personenbezeichnungen im Deutschen und im Serbischen kann festgestellt werden, dass es auch sehr viele Personenbezeichnungen gibt, die noch in keinem serbisch-deutschen Wörterbuch verzeichnet sind. Dabei geht es vor allem um expressive Personenbezeichnungen. So fehlen im Deutschen am meisten Entsprechungen für serbische Substantive mit subjektiver Einschätzung, und im Serbischen fehlen Entsprechungen für viele deutsche deonymische und metaphorische Personenbezeichnungen.

Die hier vorgetragenen Beobachtungen über Gemeinsamkeiten und Unterschiede deutscher und serbischer Personenbezeichnungen stellen keineswegs eine erschöpfende Analyse des relevanten Materials dar. Doch vielleicht könnten auch die wenigen Hinweise, vor allem auf die Unterschiede in der substantivischen Wortbildung beider Sprachen, eine Anregung sein, künftig auch die Wortbildung vor allem von expressiven Personenbezeichnungen verstärkt in kontrastiv-typologische Untersuchungen mit einzubeziehen.

\section{Literatur}

Barić, E. 1980. Imeničke složenice neprefiksalne i nesufiksalne tvorbe. Zagreb: Sveučilišna naklada Liber.

Braun, P. 1997. Personenbezeichnungen. Der Mensch in der deutschen Sprache. Tübingen: Niemeyer.

Bzdęga, A.Z. 1999. „Zusammenrückung, -setzung, -bildung“. In: Kątny, A. und Ch. Schatte. (Hrsg.). Das Deutsche von innen und außen. Ulrich Engel zum 70. Geburtstag. Poznań: Wydawnictwo Naukowe UAM. 9-23. 
Donalies, E. 2005. Die Wortbildung des Deutschen. Ein Überblick. Tübingen: Gunter Narr Verlag.

Havryliv, O. 2003. Pejorative Lexik. Untersuchungen zu ihrem semantischen und kommunikativ-pragmatischen Aspekt am Beispiel moderner deutschsprachiger, besonders österreichischer Literatur. Frankfurt am Main: Peter Lang.

Klajn, I. 2002. Tvorba reči u savremenom srpskom jeziku. Prvi deo. Slaganje i sufiksacija. Beograd: Zavod za udžbenike i nastavna sredstva.

Olsen, S. 1990. „Zum Begriff des morphologischen Heads“. Deutsche Sprache 18. 126-147.

Pittner, R. 1996. „Possessivkomposita im Neuhochdeutschen?“. In: Kunsmann, P. (Hrsg.): Linguistische Akzente 93. Beiträge zu den 3. Münchner Linguistik-Tagen. München: Verlag Dr. Kovac. 179-193.

Stanojčić, Ž. 2010. Gramatika srpskog književnog jezika. Beograd: Kreativni centar. 
